\title{
Evaluation of the Flexibility and Muscular Strength in Adult Women that Practice the Pilates ${ }^{\circledR}$ Method
}

\author{
Layana Fontes de Brito Rodrigues ${ }^{1}$, Tais Cardoso Santos ${ }^{1}$, Carlos Michell Tôrres Santos ${ }^{2}$, Gilberth Tadeu dos \\ Santos Aciole ${ }^{3}$, Jouber Mateus dos Santos Aciole ${ }^{3}$, Antônio Luiz Barbosa Pinheiro ${ }^{3}$ and Leonardo Rigoldi \\ Bonjardim $^{1}$ \\ 1. Department of Physiology, Federal University of Sergipe, São Cristovão, SE, Brazil \\ 2. Department of Physiology, Faculty of Sergipe, Aracaju, SE, Brazil \\ 3. Center of Biophotonics, School of Dentistry, Federal University of Bahia, Salvador, BA, Brazil
}

\begin{abstract}
The regular practice of physical exercises aiming the development or maintenance of flexibility and muscular strength can have a relevant role throughout life. The Pilates ${ }^{\circledR}$ method is a way of physical conditioning that strengthens and stretches the muscles without leading to exhaustion, which became popular in rehabilitation and in fitness. The present study aims to assess the benefits proposed by the Pilates ${ }^{\circledR}$ Method, among adult women that previously practiced this method as exclusive way of physical conditioning, aiming to observe if there is continuous gain of flexibility and muscular strength, and to compare these benefits within different age groups. To that matter, 20 women that practiced solely Pilates volunteered and were divided into three groups (group I: 30 to 40 years old, group II: 41 to 50 years old, group III: 51 a 60 years old). The subjects were submitted to flexibility evaluation using the Wells database strength evaluation using the 1-RM test, after 16 training sessions. The results were submitted to the One-way test ANOVA, and showed no significant differences in the gain of flexibility or strength in the three tested groups $(\mathrm{p}<0.05)$.
\end{abstract}

Key words: Physical exercise; 1-RM test; wells database.

\section{Introduction}

The Pilates method was developed by Joseph Pilates in the early 20 [1]. This technique is a form of physical conditioning that strengthens and lengthens the muscles without exhaustion, which became popular in rehabilitation and fitness. According Pilates the basis of this method is contrology, being conscious control of all the muscle movements of the body [1]. The Pilates ${ }^{\circledR}$ training is based on six principles: breathing, control, concentration, organization, articulate, smooth movement and accuracy [2].

A number of benefits is seen as improving the quality of life in practicing this technique, such as enhancement of physical conditioning and mental relief from stress-related problems, stress reduction

Corresponding author: Gilberth Tadeu dos Santos Aciole, MD, research field: physiology of the moviment. E-mail: gilberthaciole@hotmail.com. and fatigue, strength development and muscle and joint flexibility, improves posture, leading to the correct body alignment and rebalance the muscles of the trunk, which leads to the relief of chronic pain, further enhancing sports performance and general health [3].

The exercises are performed by the method covered by wood appliances, with coupled springs, which form of resistance levers that allow the development of biomechanically correct exercises [4]. In addition to the recommended exercise apparatus in use is also the working of the Ball and soil.

The maximal passive mobility of a given joint movement is defined as flexibility, which tends to be higher in females and is inversely proportional to age [6]. Muscle strength is the ability of a muscle or muscle group to generate voltage. This parameter is often measured by one repetition maximum test (1RM) [7].

The regular practice of physical exercise programs aimed at developing or maintaining flexibility and 
muscle strength can play an important role throughout life. The decline of both will gradually hindering the performance of many everyday tasks, often leads to early loss of autonomy. Therefore, adequate levels of muscular strength and flexibility are crucial for the proper functioning skeletal muscle [5]. Given the above, this study aims to evaluate the benefits offered by Pilates ${ }^{\circledR}$ in adult women who previously practiced this method as a tool for physical fitness in order to observe whether there was continued increase flexibility and strength and still compare these benefits in different age groups.

\section{Materials and methods}

\subsection{Sample}

The research was conducted in a center of Exercise Physiology in the city of Aracaju-SE. Voluntarily participated in this study 20 women divided into three groups (group I: 30 to 40 years) with mean age 34.17 years \pm 1.44 , mean weight $61.33 \mathrm{~kg} \pm 9.95$, mean height $1.66 \mathrm{~m} \pm 0.08$, (group II: 41-50 years) with mean age 44.29 years \pm 1.04 , mean weight $60.04 \mathrm{~kg} \pm 6.35$, mean height $1.61 \pm 0.01 \mathrm{~m}$; (group III: 51 to 60 years) with mean age 55.29 years \pm 1.04 , mean weight 64.14 $\mathrm{kg} \pm 6.82$, mean height $1.60 \mathrm{~m} \pm 0.04$. The proposed inclusion criteria were: age between 30 and 60 years, being female, having a practice time more than 12 months and only be practicing the Pilates method. Exclusion criteria include individuals who have musculo-skeletal pathology in a prior period of six weeks, as well as those who obtain three consecutive absences. All were informed and instructed regarding the procedures and objectives proposed by this study and gave their consents signed by a term for them.

\subsection{Stages of the Study}

The program was conducted in eight consecutive weeks, lasting 60 minutes each session, twice a week. The assessment of the flexibility and strength before starting the 16 training sessions and after completion thereof.

\subsection{Assessment of Muscular Strength}

Body flexibility was measured by the Wells Bench test used by Rebellatto et al. [8] in his study, where the measurement was performed three times and was considered a measure of greater value in $\mathrm{cm}$. In order to evaluate the muscle strength test was used for a maximum repetition (1-RM) [9], in knee extension of the lower limbs (Gevasport fitness equipment ${ }^{\circledR}$ ).

\subsection{Exercises Protocols}

We used the protocol suggested by Kolyniak et al. [1], which was adapted by inclusion and exclusion some exercises. For this, we used basically the equipaments Reformer, Trapeze, Chair Combo and Barrel (Giropilates ${ }^{\circledR}$ ), plus Ball Switzerland kinesiotherapy (Gymnic ${ }^{\circledR}$ ) 65 to $75 \mathrm{~cm}$ and ground movements.

Reformer equipments were performed in the following exercises: stretching the posterior chain in supine; Arcs and circles of the femur in the supine position, stretching the hip flexors in the foot; Strengthening of the upper limbs (biceps, triceps and rhomboids) is Lengthening the side chain trunk. In Trapeze equipment it employed the following exercises: Stretching and mobilization of the spine; Bridge with the lower limbs in extension; Strengthening of the lower limbs (quadriceps and gastrocnemius) in supine position; Strengthening of upper limbs (biceps, triceps, rotator cuff); stretching of the posterior chain. In the Combo Chair were made: Strengthening the quadriceps sitting, supine hamstring strengthening, strengthening of the upper limbs (triceps, shoulder), scapular mobilization in the prone position, stretching the posterior chain. In Barrel, their movements were developed: Stretching the posterior chain, stretching glutes and piriformis, quadriceps stretching, strengthening the lateral muscles of the trunk; strengthening abdominal muscles. On the ground, called up: Stretching the posterior chain; Bridge, circles and arcs with the femur; Serie quadruped; bearing. And finally, with the help of Swiss ball 
exercises were conducted as follows: Strengthening of the lower limbs (quadriceps and hamstrings); Bridge with knee extension and flexion; Strengthening the abdominal muscles, elongation of the spine, strengthening of the upper (biceps, triceps, shoulder, chest). Stretching contemplated initial and final support of 40 seconds, while strengthening exercises prescribed during the sessions we used two sets of 10 repetitions and stretching two sets of six repetitions.

\subsection{Statistical Analysis}

To verify the flexibility and strength differences between the groups was used analysis of variance (ANOVA-one way), since the data related to the mean and median were homogeneous. The level of significance was $\mathrm{p}<0.05$.

\section{Results}

The results obtained through the test One-way ANOVA showed no significant difference in increases flexibility and strength in the three groups studied when compared before and after 16 training sessions. The gain of flexibility, there was a weight gain of the difference between final and initial flexibility for the three groups Fig. 1.

Regarding the strength was observed an increase in average from the difference between final and initial muscular strength only to groups I and II Fig. 2.

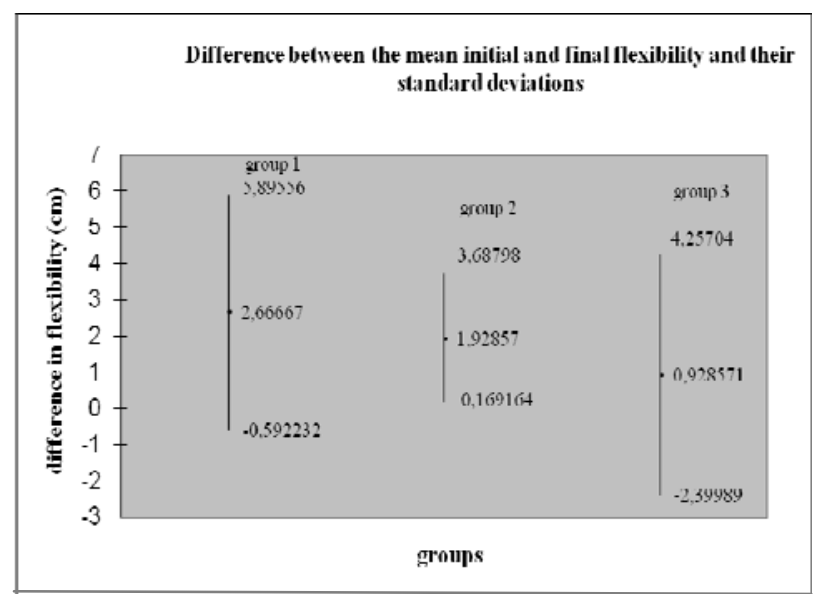

Fig. 1 Graphical representation of the confidence interval $(p<0.05)$ difference in the final and initial flexibility, as well as their standard deviations for the three groups.

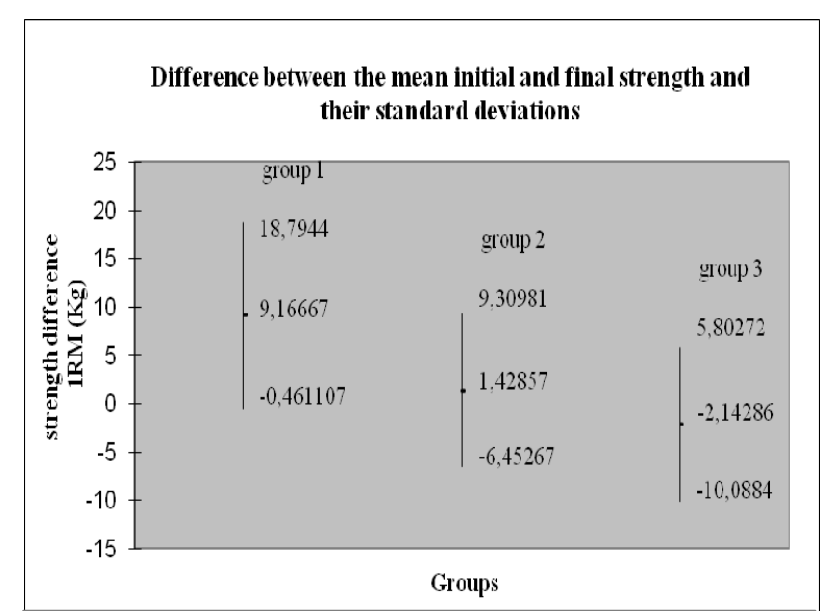

Fig. 2 Graphical representation of the confidence interval $(p<0.05)$ difference in ultimate strength and initial as well as their standard deviations for the three groups.

Denoted also that the gain of flexibility and strength vary depending on age, since the best results are shown in groups related to younger individuals and the worst results observed in the groups composed of individuals of older age.

\section{Discussion}

The variables analyzed in this study were to gain continuous posterior chain flexibility and muscle strength of lower limbs, exclusive to women practitioners of the Pilates ${ }^{\circledR}$ method for a period greater than or equal 12 months ago. However, it was not observed in the literature that examined these variables in a similar way, but several studies that observe the gain in flexibility and muscle strength as a result of the beginning of practice this training method.

The flexibility of a joint depends on your level of use. Participation in physical exercise programs can facilitate the improvement of flexibility, especially in sedentary individuals, therefore, little used joints will gradually receive a stimulus that will lead to very positive changes in the medium or long term [5]. In the present study analyzed subjects were trained in the Pilates ${ }^{\circledR}$ method 12 or more months, which therefore may have influenced the fact that he does not have a statistically significant difference between flexibility and muscle strength first and last. 
Carregaro et al [10] reports that are based on the relationship of flexibility and range of motion (ROM) joint, clinical trials are applied to assess the normality or limitation of ROM. Such events are characterized by movements that increase the distance between muscle origin and insertion, literally stretching the muscle in question in order to test it. The evaluation of the flexibility test was used to sit reach, because there is a consensus in the literature [2, 10, 12, 13], that this would be a reliable way of measuring.

Another factor that may influence the results of this study is that, according to findings Moreno et al [11], the sarcomere length is regulated by the duration of the stress the muscle is submitted: prolonged stretching can lead to a muscle adaptive process more effective compared to short periods of time.

Given the practice of Pilates ${ }^{\circledR}$, Matsudo et al. [12] in their study to analyze the muscle strength of upper and lower limbs, flexibility, agility and balance, noted that the flexibility of the lower body presented in all age groups, small increases, no statistically significant difference in the course of the year. In percentage terms this increase was $15 \%$ in the group of $50-59,6.4 \%$ in the $60-69$ and $12 \%$ in the group of $70-79$ years, confirming the results of this work.

In one recent study by Rebeltto and Castro [13], who used a practice time equal to 12 months, looking to two rest periods of activity, the results were controversial to the present work, because the women studied showed a significant gain of handgrip strength $(\mathrm{p}=0.011)$ and flexibility ( $\mathrm{p}<0.001)$ after one year of training, this difference was statistically significant from the second evaluation.

A research of 10 volunteers [14], who had before the exercise program degrees of hamstring tightness more than 30, showed that after six weeks, all volunteers had an average gain of muscle flexibility of $14.5^{\circ}( \pm 4.378)$. This finding is contrary to the results from this work, since no significant mean increase flexibility. However, it is important that this work was performed in sedentary individuals, which provides substantial gains for flexibility [5].
Silva and Farinatti [15] claim that the muscle reaches its maximum strength between the second and third decades of life and shows slow or imperceptible decline until about age 50, when it begins to decline approximately $12 \%$ to $15 \%$ per decade, with faster loss over 65 years of age. Dias et al. [16] claim that lack of familiarity with the execution of 1RM tests that are used to assess muscle strength may affect the results analysis. Such impairment may have occurred in the present study, since the participants had no familiarity with the test.

The findings of Bonci et al. [17] confirm that the interruption of an exercise program with weights produces negative effects on muscle strength. The data showed a significant decrease in the capacity of muscle strength of upper and lower limbs, especially after the eighth week of interruption. This reduction ranged from $-27.5 \%$ to $-35.1 \%$ and lower limbs to the top.

One hypothesis that could explain the difference statistically not significant force for all studied, is that second Irez et al. [18], in developing a resistance exercise session aimed at maximizing strength and hypertrophy, the first exercise of the sequence should be that you want to focus on strength gains. Since the Reformer unit was not called for the strengthening, in isolation, the quadriceps muscle and the muscle being examined in this test of strength, could have been a more substantial gains in muscular strength.

In contrast to the results presented in this work, some authors [19, 20] claim that the period of eight weeks of weight training seems to be sufficient to promote changes in muscle strength of men. Although men presented higher absolute muscle strength than women in all exercises evaluated, and women offer a more stable performance and a greater ability to resist fatigue.

\section{Conclusion}

From the present study, we conclude that the training protocol employed was able to increase flexibility and strength continuously, practicing exclusively in women's Pilates ${ }^{\circledR}$ method, however, with no 
statistically significant difference for these parameters. Suggest that further studies are performed using this technique, primarily in individuals trained in the method, since the literature discusses primarily the purpose of training in sedentary individuals. Other important aspects to focus further studies, would be to use a larger sample to perform a more targeted protocol to strengthen and gain flexibility of muscle groups to be assessed, to promote a process of familiarization with the testing of individuals, as well the use of validated tests for measuring the flexibility and strength, with the aim to elucidate all the possible application of this therapeutic modality.

\section{Acknowledgment}

The authors wish to thank the scientific and financial support given by FAPITEC/SE for the development and completion of this scientific work.

\section{References}

[1] I. E. G. G Kolyniak, S. M. B. Cavalcanti and M. S. Aoki, Isokinetic evaluation of the musculature involved in trunk flexion and extension: effect of Pilates ${ }^{\circledR}$, Brazilian Journal of Sports Medicine 10 (2004) 487-490.

[2] F. Bertolla, B. M. Baroni, E. C. P. L. Junior and J. D. Oltramari, Effect of a training program using the Pilates ${ }^{\circledR}$ in the flexibility of youth futsal athletes, Brazilian Journal of Sports Medicine 13 (2007) 222-226.

[3] Y. O. Silva, M. O. Melo, L. E. Gomes, A. Bonezi and J. F. Loss, Analysis of the external resistance and electromyographic activity of hip extension performed according to the Pilates method, Brazilian Journal of Physical Therapy 13 (1) (2009) 82-88.

[4] S. Phrompaet, A. Paungmali, U. Pirunsan and P. Sitilertpisan, Effects of pilates training on lumbo-pelvic stability and flexibility, Asian Journal of Sports Medicine 2 (1) (2011) 16-22.

[5] E. S. Cyrino, A. R. Oliveira and J. C. Leite, Behavior flexibility after 10 weeks of weight training, Brazilian Journal of Sports Medicine 10 (2004) 233-237.

[6] C. G. S. Araújo and D. S. M. S. Araújo, Flexitest: inappropriate use of condensed versions, Brazilian Journal of Sports Medicine 10 (2004) 381-384.

[7] M. I. R. Pereira and P. S. C. Gomes, Test of strength and muscular endurance: Reliability and prediction of one repetition maximum - Review and new evidence, Brazilian Journal of Sports Medicine 9 (2003) 325-335.
[8] J. R. Rebellato, J. I. Calvo, J. R. Orejuela and J. C. Portillo, Influence of a physical activity program on long-term manual muscle strength and body flexibility among elderly women, Brazilian Journal of Physical Therapy 10 (2006) 127-132.

[9] E. Unal, P. Dizmek, Y. Bilginer, A. C. Tayfur, N. Besbas and S. Ozen, The role of clinical pilates exercises in children with juvenile idiopathic arthritis: A pilot study, Pediatric Rheumatology 9 (1) (2011) 117.

[10] R. L. Carregaro, L. C. C. B. Silva and H. J. C. Gil Coury, Comparison between two clinical trials to assess the flexibility of hamstrings, Brazilian Journal of Sports Medicine 11 (2007) 139-145.

[11] M. A. Moreno, A. M. Catai, R. M. Teodori, B. L. A. Borges, M. C. César and E. Silva, Effect of a muscle stretching program for the Global Postural Reeducation method on respiratory muscle strength and thoracoabdominal mobility of sedentary young males, Braz. J. Pneumology 33 (2007) 679-686.

[12] S. M. Matsudo, V. K. R. Matsudo, T. L. B. Neto and T. L. Araújo, Evolution of neuromotor profile and functional capacity of physically active women according to chronological age, Brazilian Journal of Sports Medicine 9 (2003) 365-376.

[13] J. R. Rebellato and A. P. Castro, Effect of the revitalization program for adults on the occurrence of falls among the participants, Brazilian Journal of Physical Therapy 11 (2007) 383-389.

[14] V. R. Branco, R. F. Negrão Filho, C. R. Padovani, F. M. Azevedo, N. Alves and A. C. Carvalho, Relationship between applied voltage and the feeling of discomfort in the hamstring muscles during stretching, Brazilian Journal of Physical Therapy 10 (2006) 465-472.

[15] N. L. Silva and P. T. V. Farinatti, Influence of variables of the counter-resistance training on muscle strength in elderly people: A systematic review with emphasis on dose-response relationships, Brazilian Journal of Sports Medicine 13 (2007) 60-66.

[16] R. M. R. Dias, E. S. Cyrino, E. P. Salvador, F. L. S. Caldeira, F. Y. Nakamura and R. R. Papst, Influence of familiarization process for evaluation of muscle strength in the 1-RM, Brazilian Journal of Sports Medicine 11 (2005) 34-38.

[17] C. M. Bonci, L. J. Bonci, L. R. Granger, C. L. Johnson, R. M. Malina, L. W. Milne, R. R. Ryan and E. M. Vanderbunt, National Athletic Trainers' Association position statement: Preventing detecting, and managing disordered eating in Athletes, Journal of Athletic Training 43 (1) (2008) 80-108.

[18] G. B. Irez, R. A. Ozdemir, R. Evin, S. G. Irez and F. Korkusuz, Integrating Pilates exercise into an exercise 
program for 65+ year-old women to reduce falls, Journal of Sports Science and Medicine 10 (2011) 105-111.

[19] E. P. Salvador, E. S. Cyrino, A. L. D. Gurjão, R. M. R. Dias, F. Y. Nakamura and A. R. Oliveira, Comparison of motor performance of men and women in multiple sets of weight exercises, Brazilian Journal of Sports Medicine 11 (2005) 257-261.
[20] R. M. R. Dias, E. S. Cyrino, E. P. Salvador, F. Y. Nakamura, F. L. C. Pina and A. R. Oliveira, Impact of eight weeks of weight training on muscle strength in men and women, Brazilian Journal of Sports Medicine 11 (2005) 224-228. 